GAPP, número 23, mayo-octubre de 2020

Sección: RECENSIONES

Recibido: 13-02-2020

Modificado: 31-03-2020

Aceptado: 01-04-2020

DOI: 10.24965/gapp.i23.10777

Páginas: 167-171

\title{
SÁNCHEZ, Consuelo y RAMOS, Benito: La planificación estratégica como método de gestión pública: experiencias en la administración española
}

\section{SÁNCHEZ, Consuelo \& RAMOS, Benito: La planificación estratégica como método de gestión pública: experiencias en la administración española}

Cristina Rico Flor

Escuela Técnica Superior de Ingeniería Industrial de Barcelona (España)

ORCID: https://orcid.org/0000-0002-2734-2639

crico@andorra.ad

\section{NOTA BIOGRÁFICA}

Estudiante de doctorado del Departamento de Organización de la Escuela Técnica Superior de Ingeniería Industrial de Barcelona.

\section{RESUMEN}

Recensión: Sánchez, Consuelo y Ramos, Benito. La planificación estratégica como método de gestión pública: experiencias en la administración española. Madrid, España: Instituto Nacional de Administración Pública (INAP), 2013, 584 págs.

\section{PALABRAS CLAVE}

Gestión pública; mejora continua; planificación estratégica; gestión por objetivos; estudio de casos.

\begin{abstract}
Review: Sánchez, Consuelo \& Ramos, Benito. La planificación estratégica como método de gestión pública: experiencias en la administración española. Madrid, Spain: Instituto Nacional de Administración Pública (INAP), 2013, 584 pp.
\end{abstract}

\section{KEYWORDS}

Public management; continuous improvement; strategic planning; management by objectives; study of cases.

El objetivo del libro es exponer una metodología destinada a implementar la planificación estratégica y analizar diferentes ejemplos que se han llevado a cabo en organizaciones e instituciones que forman parte de la Administración pública española. Los autores Benito Ramos Ramos y Consuelo Sánchez Naranjo tienen una dilatada experiencia como gestores en la Administración Pública Española.

Benito Ramos Ramos es Licenciado en Ciencias Económicas y Empresariales por la Universidad Complutense de Madrid. Vinculado laboralmente a la Administración Pública Española desde 1972 su trayectoria profesional abarca diferentes ámbitos de este sector. Como formador del Instituto Nacional de Administración 
Pública (INAP) durante 17 años, en el Consejo Superior de Deportes (1988-1994), en Asuntos Sociales y Turismo (1994-1996), en gestión universitaria como Auditor Interno en la Universidad Nacional de Educación a Distancia (UNED) (1999-2002) y en gestión cultural como Secretario General del Instituto Nacional de las Artes Escénicas y de la Música (INAEM) (1996-1999) y Asesor del Secretario de Estado de Cultura (2012-2015).

Consuelo Sánchez Naranjo es Licenciada en Historia por la Universidad Autónoma de Madrid. Ingresó en el Cuerpo Superior de Administradores Civiles del Estado en 1986. Ha sido Secretaria General para la Administración Pública (2006-2007), Subsecretaria del Ministerio de Sanidad y Política Social y del Ministerio de Sanidad y Consumo (2007-2010) y desde 2012 es Asesora del Secretario de Estado de Cultura.

Los autores exponen una metodología desarrollada por la IGSAP para implementar un sistema de planificación estratégica y dirección por objetivos adaptado a las particularidades de las organizaciones administrativas, independientemente de su tamaño, objetivo y ámbito sectorial. Las aportaciones del texto resultan relevantes porque facilitan la compresión y ejecución del sistema y también porque en el campo de la planificación estratégica, prácticamente no existen guías adaptadas al sector público que tengan en cuenta las diferencias existentes con el sector privado.

Como se indica en el prólogo, el libro está dirigido a responsables de la Administración Pública que pretendan sistematizar las actividades de la organización para el logro de sus objetivos, mediante el trabajo en equipo, con criterios de eficiencia, calidad y transparencia.

Aunque la publicación data de finales del 2013, se considera que continúa estando en vigor tanto por los pocos avances que ha habido en materia de planificación estratégica para el sector público, como por los pocos cambios sustanciales que se han dado a nivel organizacional en la Administración Pública recientemente. También hay que considerar que la planificación estratégica se concibe para periodos que abarcan varios años, hecho que obliga a que las herramientas que se utilicen no tengan una caducidad inmediata y continúen siendo válidas después de varios años.

El libro se divide en dos partes que corresponden a una exposición teórica sobre el método y a la aplicación práctica de la metodología. En la primera parte de la publicación, titulada Teoría de la planificación estratégica en la administración pública se expone el método de forma detallada a lo largo de los cuatro capítulos que la componen. En la segunda parte Algunas experiencias prácticas de planificación estratégica en la Administración Pública española se presentan cinco casos prácticos de organizaciones en las que se ha aplicado la metodología. Estas abarcan diversos ámbitos como la cultura, la educación, la sanidad, la economía o el deporte y tienen modelos organizativos diferentes

En el primer capítulo, Consideraciones sobre la planificación estratégica en la administración pública, los autores justifican la aparición de la metodología explicando cómo se ha ido fraguando la necesidad de mejorar de forma significativa la gestión de la Administración Pública. Con la mejora se pretende dar respuesta a la necesidad de prestar sus servicios de forma eficiente, desde el punto de vista de la racionalización de los recursos y la transparencia.

Se debe considerar que las características organizacionales de la administración pública dificultan la implantación de la planificación estratégica como método de gestión. Aspectos tan intrínsecos como su misión constitucional, la aplicación del principio de legalidad positiva o su orientación al servicio de los intereses generales, sumado a características propias de su funcionamiento como la estructura de su dirección, la rigidez en la disponibilidad de recursos económicos, financieros y humanos, dificultan la implantación de la técnica en el sector público.

Antes de entrar a concretar la metodología, los autores dedican un apartado a definir el significado de conceptos como misión, visión, objetivo político, estrategias, proyectos, actividades, resultados e indicadores que constituyen los elementos básicos del sistema de gestión. Aunque esta nomenclatura es característica de los sistemas de gestión y su uso está generalizado, resulta útil precisar el significado adaptado a las peculiaridades de las organizaciones públicas.

A continuación, los autores presentan los cuatro módulos en los que se estructura la metodología que desarrolló la IGSAP.

En el primer módulo Incorporación al objetivo político, se analiza el objetivo político definido por los responsables de la organización. En este análisis se considera la misión y competencias de la organización, así como sus debilidades y fortalezas tanto internas como externas. A continuación, se valoran las diferentes estrategias posibles para conseguir dichos objetivos. Una vez seleccionada la estrategia más adecuada, se elabora una lista de proyectos necesarios para llevarla a cabo.

En el segundo módulo Valoración y selección de proyectos, se analizan y se seleccionan los proyectos que se consideren prioritarios o más eficaces para alcanzar el objetivo político propuesto. 
En el tercer módulo Elaboración de proyectos se define como se confeccionan los proyectos, desarrollando las actividades a realizar, asignándoles un calendario y los recursos necesarios para su realización, así como un responsable a cada una de las actividades que conforman cada proyecto. En esta fase también se determinan los resultados esperados y los indicadores que se utilizaran para medir su evolución.

En el cuarto módulo Seguimiento y evaluación se dan las pautas a observar para que los responsables de cada proyecto y los directivos de la organización puedan conocer tanto el grado de cumplimiento de los objetivos marcados como el seguimiento y evaluación del nivel de ejecución de los proyectos.

Las explicaciones se completan con unas imágenes a modo de presentación, en las que se exponen las definiciones mencionadas anteriormente y un esquema general de la metodología que se pretende implantar, con el objetivo de facilitar su entendimiento. Estas presentaciones aparecen en varias ocasiones a lo largo de la publicación y son una herramienta muy útil para comunicar conceptos de forma clara y concisa, especialmente al personal menos familiarizado con este tipo de prácticas.

A continuación, los autores abren un apartado donde explican las ventajas internas que comporta la implantación de la metodología, que se pueden resumir en sistematización de actividades, identificación de los responsables, optimización de los recursos disponibles, cohesión y motivación del equipo, transparencia y mejora en la gestión. Como consecuencia de estas explicaciones, se transmite al lector que con la aplicación de su metodología se conseguirán mayores niveles de eficiencia y eficacia en la gestión.

En cuanto a las ventajas externas, destaca la mejora de la confianza de la sociedad en los gestores de la organización, en la medida en que perciben una mejora en la eficacia, la eficiencia y la responsabilidad en la gestión que realizan. Estas reflexiones sobre las mejoras que aporta la implantación de la metodología podrían parecer triviales, pero resultan de gran ayuda cuando se necesita argumentar la utilidad del sistema a los responsables que deben tomar la decisión de implementarlo.

Los autores también exponen los inconvenientes con los que se han encontrado en su labor al implementar la metodología. Destacan la resistencia al cambio, el tiempo necesario para que el sistema madure y el peligro de creer que todos los problemas que pueda tener la organización se resolverán con la implantación del nuevo sistema de gestión. Estas reflexiones también son útiles porque ayudan a anticiparse a las dificultades que probablemente surgirán y a no crear falsas expectativas, siendo este aspecto uno de los mayores inconvenientes en la mayoría de los proyectos innovadores que se llevan a cabo en las organizaciones.

En referencia a los condicionantes y requisitos que hay que considerar antes de iniciar la implantación de la metodología, los autores identifican la implicación y compromiso de la dirección y responsables, la comunicación efectiva de los objetivos, resultados y seguimiento y la formación inicial necesaria para conseguir la perseverancia en la implantación del sistema. Tanto la comunicación como la formación son aspectos clave durante todas las fases del proceso.

En los capítulos segundo, tercero y cuarto, se indica de forma precisa como implementar la metodología.

Para facilitar la ejecución de las acciones propuestas, los autores han elaborado unas fichas para cada uno de los cuatro módulos en los que se divide el método. Cada ficha agrupa diferentes plantillas que ellos identifican como hojas. Estas doce plantillas permiten sistematizar la elaboración del plan, así como homogeneizar el sistema documental de diferentes planes estratégicos, facilitando su compresión y seguimiento.

En el primer apéndice del capítulo cuatro se presentan ejemplos prácticos con fichas elaboradas de los cuatro módulos.

En el segundo capítulo El proceso de elaboración de un plan estratégico general se ofrecen las explicaciones detalladas de cómo elaborar un plan estratégico general (PEG) adaptado a las particularidades de cada organización pública mediante la implementación del módulo 1, Incorporación al objetivo político y el módulo 2, Valoración y seguimiento de proyectos.

En el primer apartado del capítulo se explica que la realización de un PEG se modula en torno a la determinación de su Misión, Visión, Objetivos Políticos o Generales, Estrategias y Proyectos, en un horizonte temporal concreto, que suele coincidir con los periodos que duran los mandatos políticos.

En el segundo apartado se indican los dos principios metodológicos básicos que deben formar parte del proceso de elaboración de un PEG. Estos son el trabajo en equipo y el descubrimiento de lo que es necesario hacer para conseguir los objetivos, sustituyendo la visión individual por otra de conjunto bajo la corresponsabilidad en la generación de ideas desde el propio seno directivo de la organización.

En el tercer apartado de este capítulo, se presentan las indicaciones para desarrollar la implantación del módulo 1 del método, estructuradas en cuatro subapartados. En los tres primeros ámbito, fundamentos y conceptos básicos se exponen contenidos concretos. El cuarto subapartado está referido a la metodología de incorporación al objetivo y se articula en tres fases que son Examen del objetivo, Identificación de es- 
trategias y Desglose en proyectos asociados a la estrategia. Estas fases deben documentarse en las cinco hojas que aparecen a continuación, en las que se puede encontrar una explicación detallada para rellenar cada uno de sus apartados.

Las cinco hojas que forman la ficha del módulo 1 son Incorporación al objetivo, Análisis de factores estratégicos, Identificación de estrategias, Factores para la determinación de proyectos y Desglose de estrategias en proyectos posibles.

En el apartado cuatro se definen los pasos a seguir para completar el módulo 2 del método. Este apartado también se divide en cuatro subapartados, los tres primeros ámbito, fundamentos y conceptos básicos presentan información concreta. El cuarto subapartado metodología de valoración y selección de proyectos consta de dos fases que son Enumeración de los proyectos y Propuestas de proyectos. Estas fases se documentan con las hojas Lista de proyectos y Lista de proyectos propuestos por la unidad que aparecen al final del capítulo 2, en las que se puede encontrar una explicación detallada para rellenar cada uno de sus apartados

Al completar la información demandada en las siete hojas que conforman los dos primeros módulos de la metodología, se obtiene un PEG para un periodo concreto.

En el tercer capítulo El proceso de elaboración de un plan operativo anual se dan las indicaciones para poder segregar el PEG en periodos anuales y así obtener los Planes Operativos Anuales (POA).

En el primer apartado se detalla que en los POA se deben mostrar las actividades que configuran cada proyecto y las personas responsables de cada actividad, las unidades responsables de cada proyecto, los resultados que se pretenden alcanzar con su realización y los indicadores propuestos para la valoración de los resultados, durante el año que dura el plan.

En el segundo apartado se explica que el método de elaboración de un POA también se fundamenta en el trabajo en equipo de todas las personas que intervienen en los proyectos así como de los responsables de cada una de las actividades.

En el tercer apartado de este capítulo, se presentan las indicaciones para desarrollar la implantación del módulo 3 de la metodología, estructuradas en cuatro subapartados. En los tres primeros ámbito, fundamentos y conceptos básicos se exponen contenidos concretos. El cuarto subapartado está referido a la metodología de definición de proyectos, que se articula en tres fases que son Identificación, Determinación de indicadores y Descomposición en actividades.

Estas fases deben documentarse en las hojas Identificación del proyecto, Indicadores de resultados y Descripción de actividades que aparecen al final del capítulo 3, en las que se puede encontrar una explicación para rellenar cada uno de sus apartados.

En el cuarto capítulo El sistema de seguimiento y evaluación del plan operativo anual se indica como elaborar el sistema de seguimiento y evaluación del POA, que corresponde al módulo 4 de la metodología. Siguiendo las indicaciones de los autores, cabe destacar, que el sistema debe ser participativo, continuo, eficaz, objetivo, oportuno, integrado y coordinado.

En el tercer apartado de este capítulo, se presentan las indicaciones para desarrollar la implantación del módulo 4 del método, estructuradas en cuatro subapartados básicos. En los tres primeros ámbito, fundamentos y conceptos básicos se exponen contenidos concretos. Resultan especialmente útiles los conceptos sobre el análisis de desviaciones. El cuarto subapartado está referido a la metodología del seguimiento, que se articula en dos fases que son Seguimiento de la ejecución de actividades y Seguimiento de resultados. Estas fases deben documentarse en las dos hojas Seguimiento del proyecto y Seguimiento de indicadores de resultado que aparecen al final del capítulo, en las que se puede encontrar una explicación detallada para rellenar cada uno de sus apartados.

Para finalizar, los autores presentan ejemplos prácticos de cada una de las doce fichas expuestas anteriormente. Disponer de estos ejemplos, ayuda considerablemente a concretar y entender mejor el tipo de contenido que debe aparecer en cada ficha.

La elaboración de las doce plantillas propuestas permite definir una metodología de trabajo eficaz para implementar un plan estratégico realista, adaptado a las características de la organización y facilita dar seguimiento al cumplimiento de los objetivos definidos.

En la segunda parte de la publicación Algunas experiencias prácticas de planificación estratégica en la administración pública española se presentan los planes estratégicos de diferentes organismos públicos en los que se ha aplicado la metodología expuesta. Las experiencias que se presentan son el Plan Estratégico 1991-1994 del Consejo Superior de Deportes que estuvo determinado por acontecimientos como la entrada en vigor de la Ley 19/1990, del 15 de octubre, del Deporte y las Olimpiadas del 92; el Plan Estratégico 2000- 
2003 de la Universidad Nacional de Educación a Distancia que desplegó el programa electoral del rector electo; el Plan Estratégico 2002-2005 del Consejo Económico y Social que precisó de forma sistemática las ideas y proyectos del equipo directivo, el Plan Estratégico 2009-2012 de la Agencia Española de Medicamentos y Productos Sanitarios que se creó con la finalidad de transformarla en Agencia Estatal, el Plan Estratégico 2012-2015 de la Secretaria de Estado de Cultura que su elaboración coincidió con el período de una legislatura e integra dos Organismo Autónomos y dos Direcciones Generales y el Plan Estratégico 20122015 del Instituto Nacional de Administración Pública que incluyó en su publicación el primero de sus planes operativos de ejecución y cuya meta es contribuir a transformar la Administración española orientándola al ciudadano, y siendo más eficaz, trasparente, ejemplar, autónoma y responsable.

Estos ejemplos prácticos correspondientes a los planes estratégicos de organizaciones de naturaleza y funciones diversas ayudan al lector a poner en práctica las indicaciones teóricas que se dan en la primera parte de la publicación, facilitando la adecuación a su organización. También corrobora la validez y flexibilidad de la metodología para adaptarse a cualquier tipo de organización administrativa.

La experiencia acumulada en los procesos de implantación de la planificación estratégica a lo largo de un periodo de más de 20 años, confirman la utilidad y validez de la metodología.

En las experiencias mostradas en los capítulos 5,6 y 8 se encuentran a faltar ejemplos del módulo cuatro de la metodología, que corresponde a la definición de los sistemas de indicadores y que suele ser uno de los puntos donde los implantadores suelen encontrar dificultades.

En conclusión, la publicación es la exposición de una metodología desarrollada por la IGSAP que los autores, sobre la base de su dilatada experiencia, ponen al alcance del lector de forma clara y amena, y que permite aplicar los conceptos definidos específicamente para las organizaciones públicas y que se engloban en la planificación estratégica. La elaboración de los Planes Estratégicos Generales, Planes Operativos Anuales e Informes de Seguimiento y Evaluación, siguiendo las indicaciones expuestas por los autores resultarán útiles a los responsables de la implementación porque les ayudará a pasar de los conceptos teóricos a un plan de trabajo concreto, mediante la elaboración de las fichas correspondientes de cada uno de los cuatro módulos en los que se estructura la metodología.

También resuelve la dificultad inicial de por dónde empezar y cómo hacerlo, a la que se enfrentan muchos gestores cuando intentan implementar mejoras, así como concretar, de forma realista lo que se puede esperar después de adoptar la metodología.

Siendo el objetivo de la metodología conseguir la mejorar de la gestión basándose en la sistematización de las actividades orientadas al logro de sus objetivos con criterios de eficiencia, calidad y transparencia, desde el trabajo en equipo, se puede suponer que su aplicación resultará positiva, con mayor o menor intensidad según el contexto y las realidades institucionales. 